\title{
Treating breathlessness via the brain: changes in brain activity over a course of pulmonary rehabilitation
}

\author{
Mari Herigstad ${ }^{1,2,5}$, Olivia K. Faull ${ }^{1}$, Anja Hayen ${ }^{1,3}$, Eleanor Evans ${ }^{1}$, \\ F. Maxine Hardinge ${ }^{4}$, Katja Wiech ${ }^{1}$ and Kyle T.S. Pattinson ${ }^{1,4,5}$
}

Affiliations: ${ }^{1}$ FMRIB Centre, Nuffield Dept of Clinical Neurosciences, University of Oxford, Oxford, UK. ${ }^{2}$ Dept of Infection, Immunity and Cardiovascular Disease, University of Sheffield, Sheffield, UK. ${ }^{3}$ School of Psychology and Clinical Language Sciences, University of Reading, Reading, UK. ${ }^{4}$ Oxford University Hospitals NHS Foundation Trust, Oxford, UK. ${ }^{5}$ M. Herigstad and K.T.S. Pattinson should be considered joint correspondence authors.

Correspondence: Kyle Pattinson and Mari Herigstad, Nuffield Dept of Clinical Neurosciences, West Wing Level 6, John Radcliffe Hospital, Oxford, OX3 9DU, UK. E-mail: kyle.pattinsonanda.ox.ac.uk

@ERSpublications

Pulmonary rehabilitation improves breathlessness by recalibrating the brain's sensory perception networks http://ow.ly/crhy30cQerx

Cite this article as: Herigstad M, Faull OK, Hayen A, et al. Treating breathlessness via the brain: changes in brain activity over a course of pulmonary rehabilitation. Eur Respir J 2017; 50: 1701029 [https://doi.org/ 10.1183/13993003.01029-2017].

ABSTRACT Breathlessness in chronic obstructive pulmonary disease (COPD) is often discordant with airway pathophysiology ("over-perception"). Pulmonary rehabilitation profoundly affects breathlessness, without influencing lung function. Learned associations influence brain mechanisms of sensory perception. We hypothesised that improvements in breathlessness with pulmonary rehabilitation may be explained by changing neural representations of learned associations.

In 31 patients with COPD, we tested how pulmonary rehabilitation altered the relationship between brain activity during a breathlessness-related word-cue task (using functional magnetic resonance imaging), and clinical and psychological measures of breathlessness.

Changes in ratings of breathlessness word cues positively correlated with changes in activity in the insula and anterior cingulate cortex. Changes in ratings of breathlessness-anxiety negatively correlated with activations in attention regulation and motor networks. Baseline activity in the insula, anterior cingulate cortex and prefrontal cortex correlated with improvements in breathlessness and breathlessness-anxiety.

Pulmonary rehabilitation is associated with altered neural responses related to learned breathlessness associations, which can ultimately influence breathlessness perception. These findings highlight the importance of targeting learned associations within treatments for COPD, demonstrating how neuroimaging may contribute to patient stratification and more successful personalised therapy.

This article has supplementary material available from erj.ersjournals.com

Received: March 282017 | Accepted after revision: June 172017

Support statement: The study was funded by the Medical Research Council (UK) and the National Institute for Health Research Oxford Biomedical Research Centre (Oxford University Hospitals Trust). Funding information for this article has been deposited with the Crossref Funder Registry.

Conflict of interest: Disclosures can be found alongside this article at erj.ersjournals.com

Copyright CERS 2017. This ERJ Open article is open access and distributed under the terms of the Creative Commons Attribution Licence 4.0. 


\section{Introduction}

Breathlessness is an "all-consuming and life-changing" experience that is subjective, intensely personal and is associated with profound fear $[1,2]$. It is the primary symptom of chronic obstructive pulmonary disease (COPD). The severity of breathlessness is frequently discordant with airway pathophysiology [3].

The most effective treatment for breathlessness in stable COPD is pulmonary rehabilitation [4]. It benefits both personal well-being and exercise capacity, but has no measurable effect on lung function [5]. The clinical response to pulmonary rehabilitation is variable. Approximately $40 \%$ of people who complete pulmonary rehabilitation derive no measurable benefit [6].

Recently there has been a step-change in the neuroscientific understanding of sensory perception, emphasising the importance of top-down perceptual processing [2, 7]. So far, these models have been most rigorously tested in the field of visual neuroscience. A recent review by VAN DEN BERGH et al. [7] explains how such models may relate to respiratory symptom perception. Traditionally, it has been thought that peripheral sensory information is modulated by brain processes such as learning, expectation and negative affect, which influence conscious perceptions of the sensation (e.g. breathlessness) [8]. Recent models suggest that sensory perception includes a more prominent top-down process [7, 9]. Instead of being a stimulus-response organ that passively waits for ascending inputs, the brain forms predictions constructed from previous experiences (otherwise known as "priors"). These priors are then updated or corrected by neural observations of incoming sensory information [7, 9]. Negative affect, attention and interoceptive ability may act as moderators within this system, adjusting either the priors or weighting (gain) of incoming sensory information to influence symptom discordance.

Priors are generated in a stimulus valuation network comprising the anterior insula, anterior cingulate cortex, orbitofrontal cortex and ventromedial prefrontal cortex [9], which generate information and predictions about bodily state and emotion. Incoming sensory information is then fed into this network, via the periaqueductal gray and posterior insula $[10,11]$. The link between learned associations (that influence priors) and respiration has been well demonstrated in a number of experimental studies using conditioning to demonstrate how associative learning influences respiratory perception and its neural processing [10-16]).

In COPD, repeated episodes of breathlessness might reinforce learned associations with context-relevant cues (e.g. stairs, steep hills). These learned associations may then influence the brain's set of priors. Strong priors may dominate conscious perception, accompanied by reduced gain in sensory processing regions. Taken together, these processes could facilitate decoupling of symptoms from objective physiology. This decoupling is further supported by depression and anxiety [17]. Worsening breathlessness then drives a downward spiral of activity avoidance and physical deconditioning, and ever-worsening breathlessness.

Pulmonary rehabilitation interrupts this downward spiral of decline. Participants learn to cope better with their breathlessness through repeated exposure to exercise-induced breathlessness in a "safe" healthcare setting, with profound effects on the affective components of breathlessness [18, 19]. The brain mechanisms underlying this have yet to be investigated. We hypothesised that an important effect of pulmonary rehabilitation would be to reduce learned negative associations with breathlessness-related cues (i.e. a reduction in "over-perception"). This would be reflected in reduced activation in the stimulus valuation network. Extending this hypothesis, we predicted that those subjects with the strongest learned associations might have the most to gain from pulmonary rehabilitation.

We therefore assessed brain activity associated with breathlessness-related word cues in a group of patients with COPD before and after a course of pulmonary rehabilitation. Using functional magnetic resonance imaging (fMRI), we measured the brain's response to a validated set of breathlessness-related word cues [20] in combination with detailed clinical and psychological characterisation.

\section{Methods}

A brief overview of the study methods is provided here. For full details, see the supplementary material. Comparison of pre-rehabilitation fMRI findings with healthy controls and development of the breathlessness-cue task have been published elsewhere [20,21].

\section{Participants}

31 people with mild to moderate COPD (median (interquartile range) Global Initiative for Chronic Obstructive Lung Disease stage 2 (1-2), 21 male, mean \pm SD age $68 \pm 9$ years) were studied immediately preceding and following a 6-week course of outpatient pulmonary rehabilitation. All participants gave written, informed consent and the Oxfordshire Research Ethics Committee A approved the study. 


\begin{tabular}{|c|c|c|c|}
\hline & Pre-rehabilitation & Post-rehabilitation & p-value \\
\hline BMI $\mathrm{kg} \cdot \mathrm{m}^{-2}$ & $27.8 \pm 5.8$ & & \\
\hline Smoking pack-years & $40 \pm 35$ & & \\
\hline Resting $\mathrm{SaO}_{2} \%$ & $94.6 \pm 2.8$ & $94.2 \pm 3.1$ & 0.313 \\
\hline Resting heart rate beats $\cdot \min ^{-1}$ & $81.4 \pm 13.0$ & $81.4 \pm 14.2$ & 1.0 \\
\hline FEV $1 \%$ pred & $73.5 \pm 19.2$ & $75.7 \pm 29.9$ & 0.695 \\
\hline FEV $_{1} /$ FVC $\%$ & $58.0 \pm 15.9$ & $59.6 \pm 23.2$ & 0.723 \\
\hline Resting $\mathrm{PETCO}_{2} \mathrm{kPa}^{\#}$ & $4.18 \pm 0.75$ & $4.17 \pm 0.73$ & 0.92 \\
\hline
\end{tabular}

Data are presented as mean $\pm \mathrm{SD}$, unless otherwise stated. $\mathrm{BMI}$ : body mass index; $\mathrm{SaO}_{2}$ : arterial oxygen saturation; FEV1: forced expiratory volume in $1 \mathrm{~s}$; FVC: forced vital capacity; $\mathrm{PETCO}_{2}$ : end-tidal carbon dioxide tension. " : useable $\mathrm{PETCO}_{2}$ data obtained in 21 patients.

\section{Behavioural and physiological measurements and analysis}

\section{Self-report questionnaires}

The following self-report questionnaires were completed and scored according to their respective manuals: Center for Epidemiologic Studies Depression Scale (CES-D) [22], State-Trait Anxiety Inventory [23], Fatigue Severity Scale [24], St George's Respiratory Questionnaire (SGRQ) [25], Medical Research Council (MRC) breathlessness scale [26], Dyspnoea-12 (D12) questionnaire [27], Catastrophic Thinking Scale in Asthma (modified by substituting the word "breathlessness" for the word "asthma") [28], Pain Awareness and Vigilance Scale (modified by substituting the word "breathlessness" for the word "pain") [29], and Behavioural Inhibition System/Behavioural Activation System scale [30].

Physiology

Spirometry and an incremental shuttle walk test (ISWT) were undertaken according to standard practices [31].

Analysis

Full correlation matrices were calculated for all behavioural and physiological measures at baseline, and for the change following pulmonary rehabilitation, using MATLAB version R2013a (Mathworks, Natick, MA, USA). Partial correlation matrices were calculated on correlated variables, defined as $\mathrm{p}<0.05$ (uncorrected). The D12 score was taken as the clinical measure of breathlessness, and was compared with visual analogue scale (VAS) breathlessness scores both prior to and across pulmonary rehabilitation. To explore whether any of our measured behavioural variables were mediating these VAS-D12 relationships, we conducted a mediation analysis using the CANlab mediation toolbox [32].

\begin{tabular}{|c|c|c|c|}
\hline & Pre-rehabilitation & Post-rehabilitation & p-value \\
\hline MRC score & $2.9 \pm 0.8$ & $2.7 \pm 0.9$ & 0.231 \\
\hline Dyspnoea-12 & $12.0 \pm 9.2$ & $7.9 \pm 6.4$ & 0.009 \\
\hline SGRQ & $49.9 \pm 17.4$ & $42.7 \pm 14.2$ & 0.001 \\
\hline Catastrophic Thinking Scale & $12.1 \pm 10.3$ & $7.2 \pm 5.4$ & 0.022 \\
\hline Awareness and Vigilance Scale & $40.5 \pm 13.0$ & $39.0 \pm 14.2$ & 0.514 \\
\hline Fatigue Severity Scale & $41.4 \pm 11.3$ & $32.6 \pm 11.6$ & 0.001 \\
\hline CES-D & $13.3 \pm 8.9$ & $11.8 \pm 7.3$ & 0.375 \\
\hline State anxiety & $35.1 \pm 9.1$ & $32.3 \pm 9.4$ & 0.150 \\
\hline Trait anxiety & $35.8 \pm 9.1$ & $31.9 \pm 8.8$ & 0.010 \\
\hline BIS/BAS & $54.0 \pm 7.4$ & $53.9 \pm 7.2$ & 0.906 \\
\hline ISWT m & $342 \pm 199$ & $426 \pm 212$ & 0.000001 \\
\hline \multicolumn{4}{|c|}{$\begin{array}{l}\text { Data are presented as mean } \pm S D \text {, unless otherwise stated. Behavioural measurements pre- and } \\
\text { post-pulmonary rehabilitation ( } n=31 \text { ). MRC: Medical Research Council breathlessness scale; SGRQ: St } \\
\text { George's Respiratory Questionnaire; CES-D: Center for Epidemiologic Studies Depression Scale; BIS/BAS: } \\
\text { Behavioural Inhibition System/Behavioural Activation System scale; ISWT: incremental shuttle walk test. }\end{array}$} \\
\hline
\end{tabular}



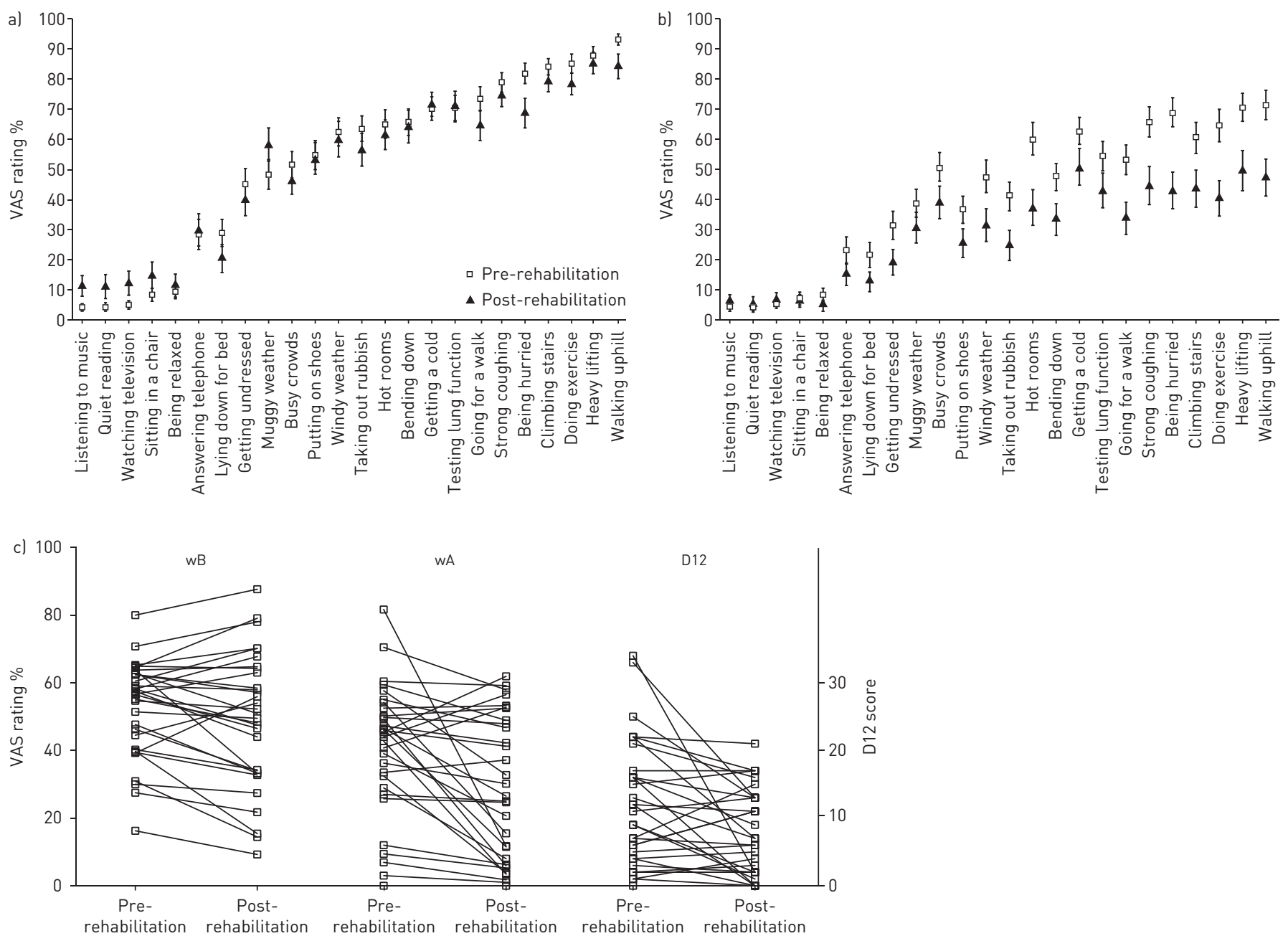

FIGURE 1 Demonstration of changes in visual analogue scales (VASs) relating to a) "How breathless would this make you feel?" (wB) and b) "How anxious would this make you feel" (wA), and c) individual changes in wB, wA and Dyspnoea-12 (D12) score over the course of pulmonary rehabilitation.

\section{Brain imaging and analysis}

MRI was performed with a Siemens $3 \mathrm{~T}$ TIM-Trio scanner (Siemens, Camberley, Surrey, UK), using a 12-channel head coil. A structural T1-weighted scan (voxel size $1 \times 1 \times 1 \mathrm{~mm}$ ), fMRI T2*-weighted scan (voxel size $3 \times 3 \times 3 \mathrm{~mm}$ ) and fieldmaps were collected. During fMRI scanning, participants were shown a set of breathlessness-related word cues [20], and asked to rate each cue according to breathlessness and breathlessness-anxiety on a VAS with the question "How breathless would this make you feel?" (wB) and the "How anxious would this make you feel?" (wA). Word cues were chosen that were applicable to all subjects. All subjects viewed the same set of words during both sessions, with the order of word presentation randomised within each session.

A control condition of matched random-letter-string presentations was interspersed with the breathlessness cues. This allowed us to test whether any changes in mean VAS responses related to generalised changes in negative valence or arousal, or were more breathlessness specific.

fMRI data processing was carried out within FSL (Oxford Centre for Functional Magnetic Resonance Imaging of the Brain Software Library, Oxford, UK), using FEAT (fMRI Expert Analysis Tool version 5.98). The cluster $Z$ threshold was 2.3 and the corrected cluster significance threshold $\mathrm{p}=0.05$ [33]. Analysis was corrected for multiple comparisons across the whole brain; region-of-interest approaches were not used.

The first-level (individual subject) analysis in FEAT incorporated a general linear model, with explanatory variables included for word presentation, and two (de-meaned) explanatory variables modelling the trial-by-trial variability in VAS responses to the breathlessness (wB) and breathlessness-anxiety (wA) cues 

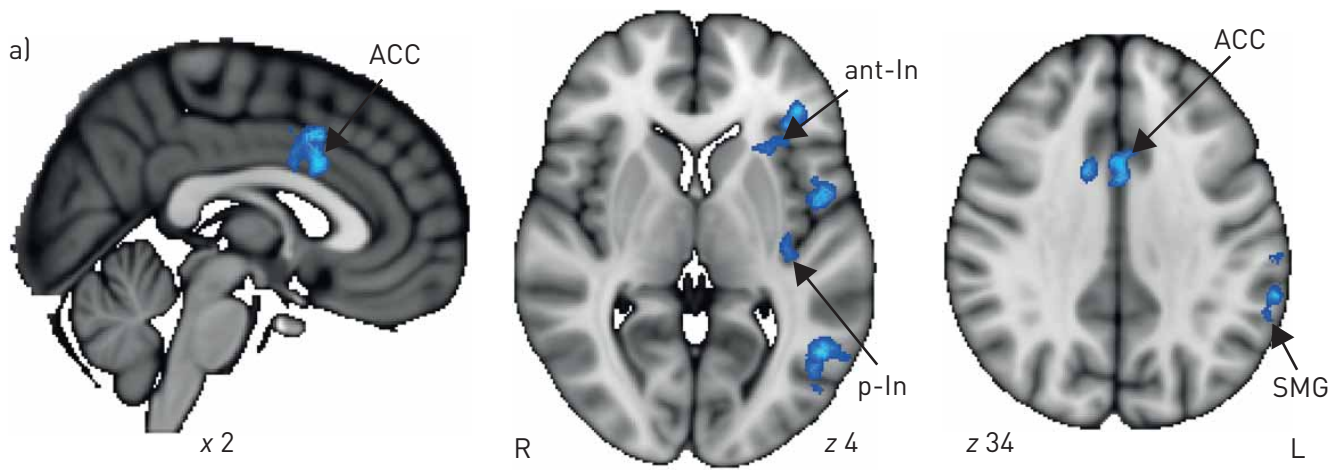

b)

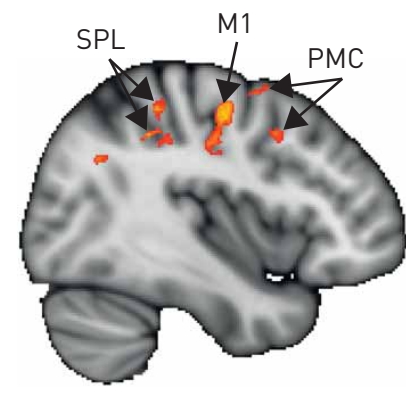

$\times 40$

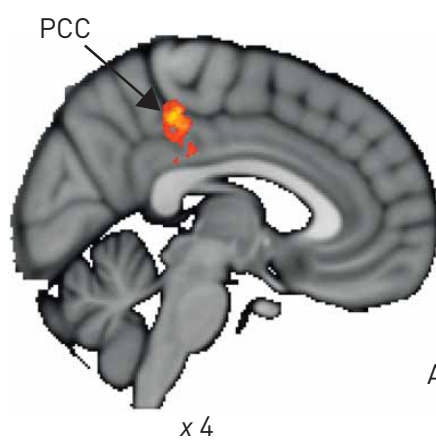

4.0; decreased brain activity Z-score 2.3

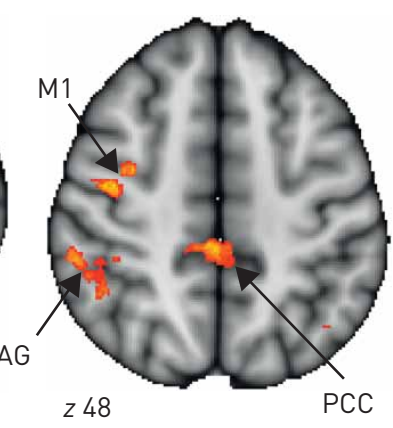

4.0

FIGURE 2 Change in blood oxygen level-dependent activity that correlates with rehabilitation-related changes in behavioural scores of breathlessness response to al "How breathless would this make you feel?" (wB) (blue-light blue) and b) "How anxious would this make you feel?" (wA) (red-yellow). ACC: anterior cingulate cortex; ant-In: anterior insula; p-In: posterior insula; R: right; SMG: supramarginal gyrus: L: left; SPL: superior parietal lobe; M1: primary motor cortex; PMC: premotor cortex; PCC: posterior cingulate cortex; AG: angular gyrus. The images consist of a colour-rendered statistical map superimposed on a standard (Montreal Neurological Institute $1 \times 1 \times 1 \mathrm{~mm}$ ) brain; significant regions are displayed with a threshold $Z>2.3$, with a cluster probability threshold of $p<0.05$ (corrected for multiple comparisons).

presented during scanning. A middle-level analysis was then conducted to calculate a difference in brain activity from pre- to post-rehabilitation for each subject.

The group-level analysis aimed to interrogate which brain regions could account for between-subject variability in the VAS responses over the course of pulmonary rehabilitation. To do this, we regressed each individual's change in $\mathrm{wB}$ and $\mathrm{wA}$ VAS scores against the change in mean word-cue explanatory variable from the middle-level analysis. We also conducted a secondary analysis, where we regressed the change in VAS responses on pre-rehabilitation word-cue brain activity, to probe baseline factors associated with improved breathlessness following pulmonary rehabilitation. Mediation analyses were also conducted on the relationship between VAS score and correlated brain regions, with the behavioural questionnaire scores as potential mediators, using the CANlab mediation toolbox [32].

\section{Results}

\section{Participants}

Demographic, physiological and questionnaire data, as well as averaged breathlessness VAS ratings, are presented in tables 1 and 2. Improvement above the minimum clinically important difference (MCID) in quality of life (as measured by SGRQ [34]) occurred in 16 subjects (52\%; MCID 4 points), functional exercise capacity (as measured by ISWT [35]) in 19 subjects (61\%; MCID $-48 \mathrm{~m}$ ) and clinical measures of breathlessness (as measured by D12 [36]) in 14 subjects (45\%; MCID 3 points).

Pulmonary rehabilitation led to a group mean \pm SD improvement in wA VAS responses (pre $40.5 \pm 19.4$ versus post $28.8 \pm 21.1 ; \mathrm{p}=0.002$, two-tailed paired t-test). As illustrated in figure 1 , over the course of pulmonary rehabilitation, in 24 patients wA decreased, in six patients wA increased and in one patient there was no change. Improvements in $\mathrm{wB}$ VAS response did not reach statistical significance (pre $52.6 \pm 14.2$ versus post 49.1 $\pm 19.9 ; \mathrm{p}=0.086$ two-tailed paired $\mathrm{t}$-test). Over the course of pulmonary rehabilitation, in 22 patients $w \mathrm{~B}$ decreased and in nine patients $\mathrm{wB}$ increased. 
a)

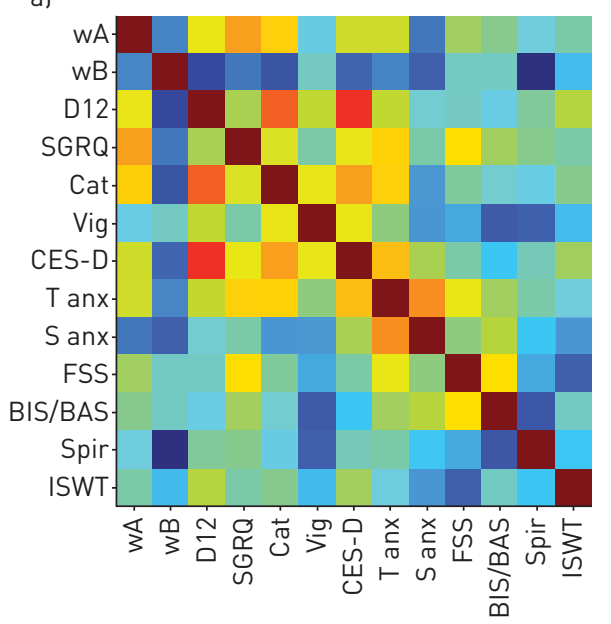

b)

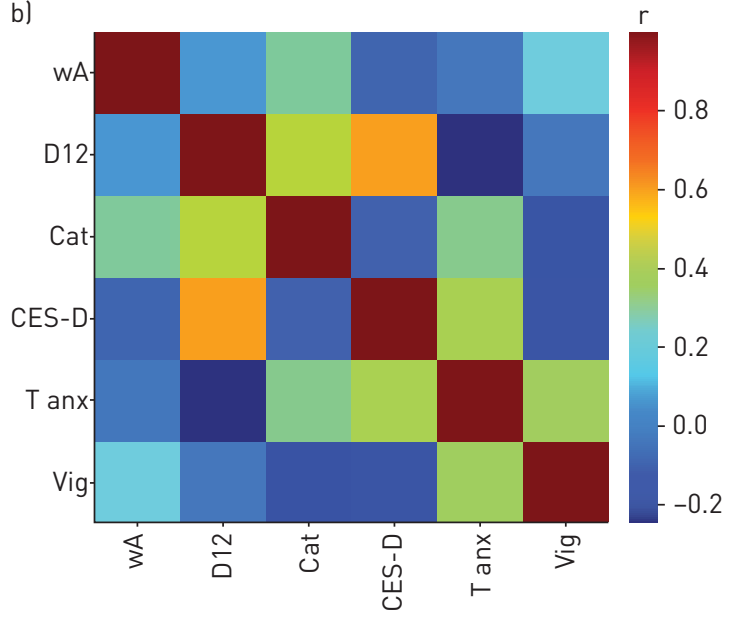

FIGURE 3 Exploratory analyses demonstrating correlations of changes in the measured variables across pulmonary rehabilitation: a) full correlation matrix of all the measured behavioural variables and b) partial correlation matrix of those variables that significantly correlated with Dyspnoea-12 (D12) score (defined at $\mathrm{p}<0.05$, uncorrected). wA: visual analogue scale (VAS) "How anxious would this make you feel?"; wB: VAS "How breathless would this make you feel?"; SGRQ: St Georges Respiratory Questionnaire; Cat: breathlessness catastrophising score; Vig: breathlessness vigilance and awareness score; CES-D: Center for Epidemiologic Studies Depression Scale; T anx: trait anxiety; S anx: state anxiety; FSS: Fatigue Severity Scale; BIS/BAS: Behavioural Inhibition System/Behavioural Activation System scale; Spir: spirometry lforced expiratory volume in $1 \mathrm{~s} /$ forced vital capacity); ISWT: incremental shuttle walk test. The partial correlations are independent of the other factors within this matrix only. The SGRQ and ISWT were excluded from the partial correlation despite significant correlation, as they are summary measures combining various psychological constructs.

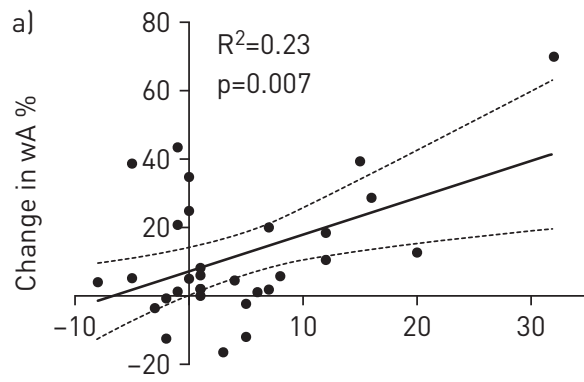

Change in D12 AU

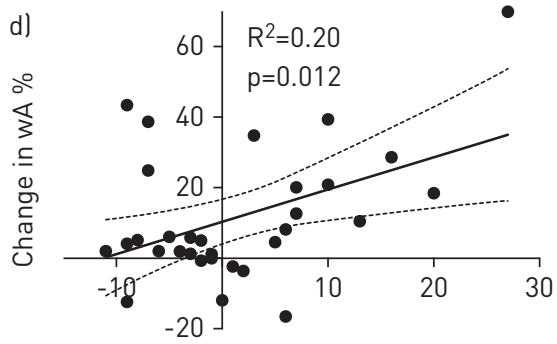

Change in CES-D AU b)

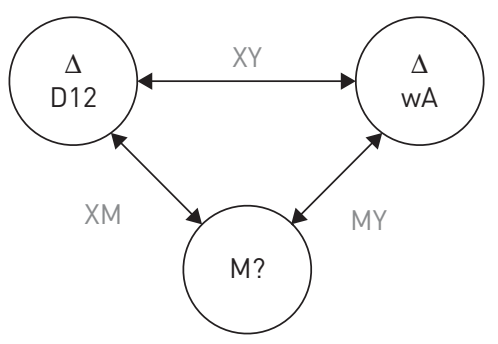

e)

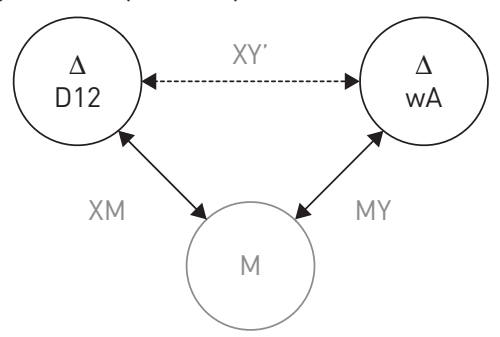

c)
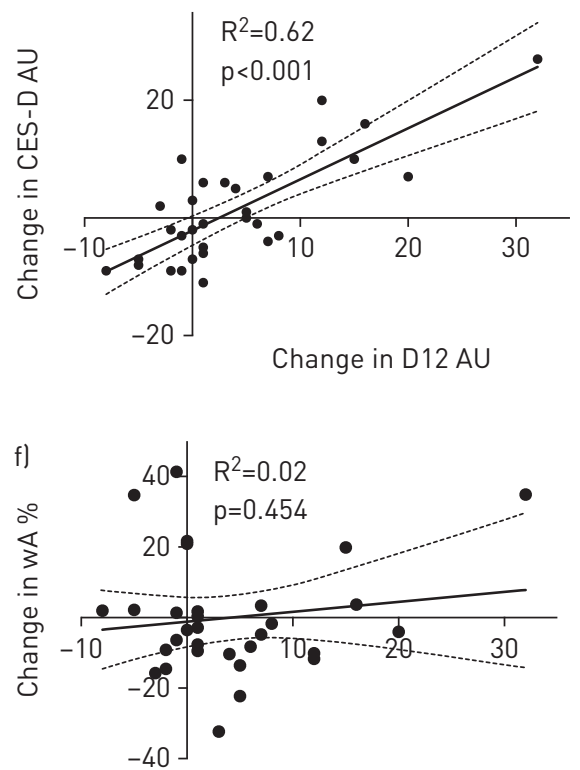

Change in D12 AU

FIGURE 4 Mediation analysis: relationship between change in word-cue breathlessness-anxiety (wA) and change in Dyspnoea-12 (D12) score across the course of pulmonary rehabilitation, mediated by change in depression score. AU: arbitrary units; CES-D: Center for Epidemiologic Studies Depression Scale. a) Correlation between change in visual analogue scale (VAS) score for wA and change in D12 score. b) Depiction of a mediation, where the relationship between change $(\Delta)$ in wA and D12 (XY) can be mediated by $M$, which correlates with both X (D12; XM) and $Y$ (wA; XY). c) XM relationship: X variable (D12) predicts mediator variable (depression). d) MY relationship: mediator variable (depression) predicts $Y$ variable (wA). e) Depiction of residual relationship ( $\left.X Y^{\prime}\right)$ once the effect of mediator variable $M$ has been removed. $f$ ) Residual relationship between change in wA and change in D12 when change in depression has been regressed out. For full explanation of mediation, see the supplementary material. 

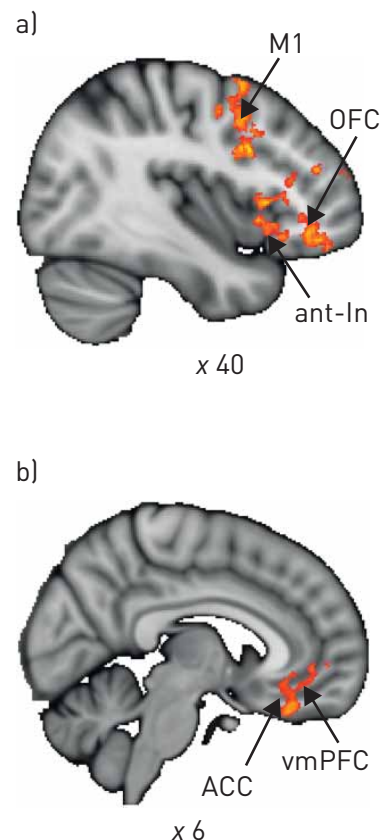
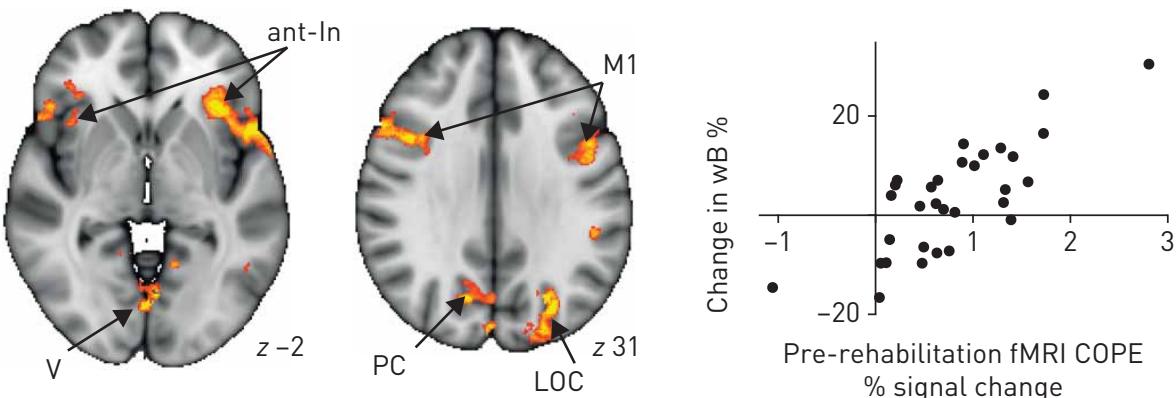

Pre-rehabilitation $\mathrm{FMRI}$ COPE $\%$ signal change

Larger pre-rehabilitation activity correlating with post-rehabilitation vmPFC

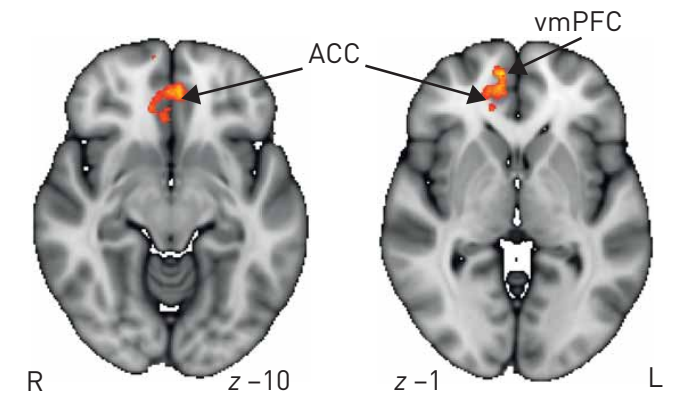

$\mathrm{L}$

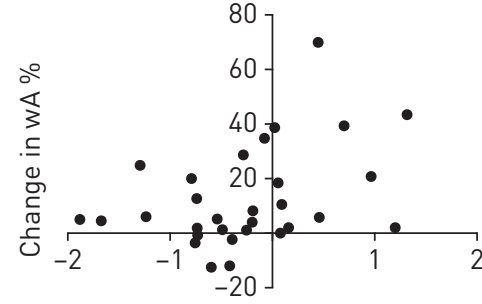

Pre-rehabilitation fMRI COPE

$\%$ signal change decrease in behavioural Z-score $2.3 \quad 4.0$

FIGURE 5 Pre-rehabilitation blood oxygen level-dependent activity that correlates with rehabilitation-related changes in behavioural scores of breathlessness response to a) "How breathless would this make you feel?" (wB) and b) "How anxious would this make you feel" (wA). M1: primary motor cortex; OFC: orbitofrontal cortex; ant-In: anterior insula; V: visual cortex; PC: precuneus cortex; LOC: lateral occipital cortex; fMRI: functional magnetic resonance imaging; COPE: contrast of parameter estimate; ACC: anterior cingulate cortex; vmPFC: ventromedial prefrontal cortex; R: right; L: left. The images consist of a colour-rendered statistical map superimposed on a standard (Montreal Neurological Institute $1 \times 1 \times 1 \mathrm{~mm}$ ) brain; significant regions are displayed with a threshold Z>2.3, with a cluster probability threshold of $p<0.05$ (corrected for multiple comparisons). The graphs represent linear regression between the averaged COPE value in the significant regions and the change in wB and $\mathrm{WA}$ scores, respectively.

\section{Variability of response to pulmonary rehabilitation}

Here, we present the findings of the longitudinal repeated measures analysis examining the changes in neural responses and behaviour associated with changes in breathlessness-related word cues over the course of pulmonary rehabilitation (figure 2).

\section{"How breathless would this make you feel?" (wB)}

Changes in $\mathrm{wB}$ ratings over the course of pulmonary rehabilitation were positively correlated with changes in blood oxygen level-dependent (BOLD) activation in the left anterior insula, left posterior insula, left supramarginal gyrus and anterior cingulate cortex. We did not observe any significant activations in the opposite direction.

\section{"How anxious would this make you feel?" (wA)}

Changes in wA ratings over the course of pulmonary rehabilitation were negatively correlated with changes in BOLD activity in the posterior cingulate cortex, angular gyrus, primary motor cortex and supramarginal gyrus. We did not observe any significant activations in the opposite direction.

\section{Random letter strings}

No changes in brain activity to random letter strings that correlated with changes in either wB or wA were observed.

Behavioural changes with pulmonary rehabilitation

Exploratory full and partial correlation matrices of all behavioural scores are illustrated in figure 3.

\section{Mediation analysis}

A mediation analysis incorporated all variables [32] to investigate the relationship between changes in wA and $\mathrm{wB}$, and their relationship to change in D12 (our clinically validated measure). Change in $\mathrm{wB}$ was not 
correlated with D12, so mediation did not proceed further. The relationship between wA and D12 was significantly mediated by change in depression, and the residuals (once depression was accounted for) were nonsignificant (figure 4).

\section{Baseline factors associated with improved breathlessness following pulmonary rehabilitation} Here, we present the findings of an analysis that examines how baseline brain activity correlates with changes in the behavioural responses to breathlessness-related word cues over the course of pulmonary rehabilitation (figure 5).

\section{"How breathless would this make you feel?" (wB)}

We observed a positive correlation between change in $\mathrm{wB}$ and pre-rehabilitation activity in the anterior insula (bilateral), orbitofrontal cortex (bilateral), precuneus, lateral occipital cortex and primary motor cortex (bilateral).

"How anxious would this make you feel?" (wA)

We observed a positive correlation between change in wA and pre-rehabilitation activity in the anterior cingulate cortex and ventromedial prefrontal cortex.

\section{Discussion}

Key findings

Pulmonary rehabilitation was associated with improvements in breathlessness, exercise capacity and quality of life, in line with previously published research $[4,6,18,19]$. Changes in ratings of VAS breathlessness (wB) positively correlated with changes in activity in the brain's stimulus valuation network, whereas changes in ratings of VAS breathlessness-anxiety (wA) negatively correlated with activity in attentionregulating networks. At baseline, activity in the stimulus valuation network correlated with change in $w B$ and wA scores following pulmonary rehabilitation.

\section{Effect of pulmonary rehabilitation on brain and behaviour}

Group improvements were observed in a range of affective domains (table 2) that occurred independently of any changes in spirometry [5]. However, not all participants showed clinically important improvements, emphasising the need to understand the brain processes that are associated with pulmonary rehabilitation.

In chronic diseases such as COPD, prior experiences of symptoms can vastly alter outcomes and symptom perception [37]. Learned associations between environmental cues and symptoms can influence this symptom expectation and subsequent perception. We therefore used a word-cue task to probe these associations. The changes in ratings of breathlessness-anxiety (wA) positively correlated with the changes in the clinical measure of breathlessness (D12) across the course of rehabilitation (figure 4). This supports previous work demonstrating that pulmonary rehabilitation has greater effects upon the affective components of breathlessness [18, 19].

We then examined the changes in brain activity associated with word-cue presentation across pulmonary rehabilitation. Changes in the ratings of breathlessness $(\mathrm{wB})$ were positively correlated with changes in brain activity in the anterior insular cortex, anterior cingulate cortex, prefrontal cortex and posterior insular cortex. Taken together, these form key components of the stimulus valuation network, which is responsible for the conscious awareness of the internal state of the body, or interoception [9]. These observations may represent re-evaluated associations relating to breathlessness expectations.

The posterior insula plays a role in sensory-discriminative processing, with connectivity both to somatosensory cortices and to the anterior insula to integrate sensory inputs with the stimulus valuation network [9]. The positive correlation between changes in $\mathrm{wB}$ ratings and changes in BOLD activity seen in this structure over the course of pulmonary rehabilitation suggests that the reappraisal of learned associations may also influence lower-order sensory processing.

fMRI activity was negatively correlated with ratings of breathlessness-anxiety (wA) in the angular gyrus, supramarginal gyrus and posterior cingulate cortex. These changes in brain activity could represent a shift in expectancy towards that seen in healthy controls $[14,21]$. The angular gyrus and supramarginal gyrus form a multimodal complex that integrates somatosensory inputs to the brain and is associated with attention processing. The posterior cingulate cortex is thought to mediate interactions between emotions and memory, controlling attentional focus [38]. All these functions are impaired by anxiety [39, 40]. Additionally, the increase in activity in the granular primary and pre-motor cortices may reflect a shift towards more objective perceptual processing less dominated by learned associations $[9,14,41]$. 
Baseline fMRI correlates with changes in VAS scores following pulmonary rehabilitation

Change in VAS responses over pulmonary rehabilitation was positively correlated with baseline BOLD activity in the ventromedial prefrontal cortex and anterior cingulate cortex in response to wA ratings (figure 5), and in the anterior insula (bilateral), orbitofrontal cortex and motor cortex in response to $\mathrm{wB}$ ratings. These areas comprise much of the stimulus valuation network. Our findings suggest that people with greater brain activity during word-cue presentation are more likely to benefit from pulmonary rehabilitation, particularly with regard to the wA contrast, as this correlates with the clinically validated D12 score. Although tempting to dissect the specific brain areas relating to $\mathrm{wA}$ and $\mathrm{wB}$ in more detail, this is best reserved for future studies where connectivity profiles might better explain the components of this network function more comprehensively.

It is possible that the repeated episodes of breathlessness experienced in a "safe" setting during pulmonary rehabilitation help to re-shape associations. These findings and interpretations are supported by a recent behavioural study [42], which showed that higher breathlessness-fear before pulmonary rehabilitation was associated with greater reductions in breathlessness during exercise following pulmonary rehabilitation.

\section{Driving forces behind changes in behaviour}

We also interrogated how the relationship between VAS scales and associated brain activity may be related to other clinical and behavioural measures of breathlessness. The full correlation matrix (figure 3) demonstrates that many of the behavioural measures are at least partially explained by each other. A notable exception is spirometry, which is poorly explained by all of the other measures. This distinction between lung function and the various measures of breathlessness perception adds to the weight of evidence that the impact of COPD is poorly reflected by spirometry [3].

When investigating the VAS scores associated with the breathlessness expectancy task, the strongest correlation with wA was the D12 questionnaire; a well-validated clinical measure of breathlessness [27]. In addition, strong correlations exist between wA and breathlessness catastrophising and depression. Conversely, the $\mathrm{wB}$ measure did not correlate significantly with any of the measures. Mediation analysis [32] revealed that the relationship between $\mathrm{WA}$ and the D12 scale appears to be mediated by changes in depression, and when this component is removed there is no remaining relationship between D12 and wA (figure $4 \mathrm{f}$ ). Although the direction of the relationship between anxiety, depression and breathlessness remains unknown, many consider it to be bidirectional [17]. Some even speculate that aberrant predictions mediated by the stimulus valuation network may drive depression, anxiety and fatigue [9].

Thus, based upon predictive models of sensory perception, we speculate that pulmonary rehabilitation exerts its benefit by two broad mechanisms in the brain: 1) by updating of the brain's set of breathlessness-related priors (through associative learning), and 2) by acting upon measures of negative affect that act as moderators of priors and influence gain of sensory information processing. Improvements in muscle function with fitness would additionally adjust afferent inputs [4]. These factors are likely to work together to contribute to the well-documented improvements in well-being with pulmonary rehabilitation $[4,19,42]$.

\section{Further considerations}

Although some of the brain regions identified in the present study overlap with brain regions activated by induced breathlessness, it is important to note that this does not necessarily implicate activity in identical neuronal populations or networks. A single MRI voxel may contain over half a million neurons, not all of which would activate simultaneously. Future work could also explore connectivity profiles, or functional communication between different areas of the brain, which may differ across perceptions even with similar task activation patterns.

Although it is possible that changes in ratings of breathlessness (wB) and breathlessness-anxiety (wA) may be influenced by a generalised changes in negative valence, arousal or attention, the fact that we observed no changes in brain activity correlated with $\mathrm{wB}$ and $\mathrm{wA}$ during the random-letter-string control task suggests that this unlikely to be a major confound.

The current study used a parametric design, investigating changes in brain activity that correlated with individual subject's VAS scores of $\mathrm{wA}$ and $\mathrm{wB}$. With the range of responses to pulmonary rehabilitation, future work could look to more formally compare responders with nonresponders, but this would require considerably larger sample sizes.

We did not include a control group of COPD subjects who either did not undertake pulmonary rehabilitation treatment or received a sham treatment. Without a control group, potential contributing factors (such as placebo effects and natural fluctuations in disease severity) cannot be disentangled from the effects of pulmonary rehabilitation. However, our primary question was to understand the brain 
mechanisms underlying subject-specific changes in breathlessness perception. Future work may wish to include a control group, to better understand group-wide effects of rehabilitation compared with control or sham-treated subjects. Importantly, the clinical changes we have observed are very much in line with clinical reports of pulmonary rehabilitation (e.g. the UK National Pulmonary Rehabilitation Audit [6]), suggesting we have studied a representative sample of individuals.

\section{Clinical relevance}

This study examined brain mechanisms underlying the variable changes in breathlessness over a course of pulmonary rehabilitation. We have shown that changes in breathlessness ratings are positively correlated with changes in activity in the stimulus valuation network, and changes in ratings of breathlessness-anxiety are negatively correlated with activity in attention processing and motor control areas of the brain. We have also demonstrated that changes in ratings of breathlessness-anxiety correspond with a clinically meaningful measure of breathlessness, the D12 questionnaire. Therefore, specifically targeting associative learning might be a way to further improve efficacy of pulmonary rehabilitation. This might arise from combining pulmonary rehabilitation with drugs that target relevant neurotransmitter systems [43]. As many patients decline the invitation to pulmonary rehabilitation, alternative behavioural therapies could be developed to focus on re-evaluating the interpretation of respiratory sensations, such as through breathing exercises or mindfulness [44].

Our findings also demonstrate the first steps towards using fMRI as a tool for patient stratification. We speculate that this is most likely to be achieved in real life by using a detailed understanding of neural mechanisms to guide the development of appropriate behavioural tests (questionnaires, computerised tasks) that can be used at the bedside. These tests could be incorporated with appropriate clinical measures to personalise the treatment of COPD, targeting treatment options where they are needed in each individual.

\section{Conclusions}

In conclusion, we have demonstrated that changes in ratings of breathlessness and breathlessness-anxiety over the course of pulmonary rehabilitation likely reflect changes in associative learning. Our findings suggest that changes in the brain responses to breathlessness-related word cues over the course of pulmonary rehabilitation become less dependent upon learned associations, thus reducing "over-perception" of symptoms. Understanding the neural processing of breathlessness in a clinical population is crucial for advances to be made in its treatment, such as the development of patient stratification, leading to individualised treatments that may target breathlessness independently of disease mechanisms.

\section{Acknowledgements}

The authors thank Steve Knight, Bethan Hughes, Isabel Chabata, Debby Nicoll, Tara Harris, Fran Sinfield, Emma Tucker, Rachel Lardner, Julie Young and the Oxfordshire Pulmonary Rehabilitation Team (Oxford, UK) for their continued support of the study, and Richard Wise (Cardiff, Cardiff, UK), Andrea Reinecke (University of Oxford, Oxford, UK), Rob Davies (deceased; formerly University of Oxford), Annabel Nickol (University of Oxford) and Najib Rahman (University of Oxford) for their generous input. We wish to thank Caroline Jolley (King's College London, London, UK), Sara Abdallah (McGill University, Montreal, QC, Canada), Sara Booth (University of Cambridge, Cambridge, UK) and Ben Ainsworth (Southampton University, Southampton, UK) for their comments on previous versions of this manuscript.

Author contributions: M.H., O.K.F and K.T.S.P. had full access to all the data in the study, and take responsibility for the integrity of the data and the accuracy of the data analysis. M.H., F.M.H., K.W. and K.T.S.P. contributed to the study concept and design. M.H., O.K.F., A.H., E.E., K.W. and K.T.S.P. contributed to the data acquisition and analysis. All authors contributed to drafting the manuscript and reviewing it for important intellectual content. All authors contributed to the final approval of the manuscript.

\section{References}

Carel H. Illness: The Cry of the Flesh. Stocksfield, Acumen, 2008.

2 Hayen A, Herigstad M, Pattinson KT. Understanding dyspnea as a complex individual experience. Maturitas 2013; 76: $45-50$.

3 Parshall MB, Schwartzstein RM, Adams L, et al. An official American Thoracic Society statement: update on the mechanisms, assessment, and management of dyspnea. Am J Respir Crit Care Med 2012; 185: 435-452.

4 McCarthy B, Casey D, Devane D, et al. Pulmonary rehabilitation for chronic obstructive pulmonary disease. Cochrane Database Syst Rev 2015; 2: CD003793.

5 Carrieri-Kohlman V, Donesky-Cuenco D, Park SK, et al. Additional evidence for the affective dimension of dyspnea in patients with COPD. Res Nurs Health 2010; 33: 4-19.

6 National COPD Audit Programme. Pulmonary rehabilitation: steps to breathe better. National Chronic Obstructive Pulmonary Disease (COPD) Audit Programme: clinical audit of pulmonary rehabilitation services in England and Wales 2015. 2016. www.rcplondon.ac.uk/projects/outputs/pulmonary-rehabilitation-steps-breathebetter Date last accessed: July 21, 2017. 
7 Van den Bergh O, Witthoft M, Petersen S, et al. Symptoms and the body: taking the inferential leap. Neurosci Biobehav Rev 2017; 74: 185-203.

8 Lansing RW, Gracely RH, Banzett RB. The multiple dimensions of dyspnea: review and hypotheses. Respir Physiol Neurobiol 2009; 167: 53-60.

9 Barrett LF, Simmons WK. Interoceptive predictions in the brain. Nat Rev Neurosci 2015; 16: 419-429.

10 Faull $\mathrm{OK}$, Jenkinson $\mathrm{M}$, Ezra $\mathrm{M}$, et al. Conditioned respiratory threat in the subdivisions of the human periaqueductal gray. Elife 2016; 5: e12047.

11 Faull OK, Pattinson KT. The cortical connectivity of the periaqueductal gray and the conditioned response to the threat of breathlessness. Elife 2017; 6: e21749.

12 Pappens M, Vandenbossche E, Van den Bergh O, et al. Interoceptive fear learning to mild breathlessness as a laboratory model for unexpected panic attacks. Front Psychol 2015; 6: 1150.

13 Van Diest I, De Peuter S, Piedfort K, et al. Acquired lightheadedness in response to odors after hyperventilation. Psychosom Med 2006; 68: 340-347.

14 Hayen A, Wanigasekera V, Faull OK, et al. Opioid suppression of conditioned anticipatory brain responses to breathlessness. Neuroimage 2017; 150: 383-394.

15 Faull OK, Hayen A, Pattinson KTS. Breathlessness and the body: neuroimaging clues for the inferential leap. Cortex 2017; in press. [https://doi.org/10.1016/j.cortex.2017.07.019]

16 Stoeckel MC, Esser RW, Gamer M, et al. Brain responses during the anticipation of dyspnea. Neural Plast 2016; 2016: 6434987.

17 von Leupoldt A, Kenn K. The psychology of chronic obstructive pulmonary disease. Curr Opin Psychiatry 2013; 26: $458-463$.

18 Carrieri-Kohlman V, Gormley JM, Eiser S, et al. Dyspnea and the affective response during exercise training in obstructive pulmonary disease. Nurs Res 2001; 50: 136-146.

19 Wadell K, Webb KA, Preston ME, et al. Impact of pulmonary rehabilitation on the major dimensions of dyspnea in COPD. COPD 2013; 10: 425-435.

20 Herigstad M, Hayen A, Reinecke A, et al. Development of a dyspnoea word cue set for studies of emotional processing in COPD. Respir Physiol Neurobiol 2016; 223: 37-42.

21 Herigstad M, Hayen A, Evans E, et al. Dyspnea-related cues engage the prefrontal cortex: evidence from functional brain imaging in COPD. Chest 2015; 148: 953-961.

22 Radloff L. A self-report depression scale for research in the general population. Appl Psychol Meas 1977; 1: 385-401.

23 Spielberger CD, Gorsuch RL, Lushene R, et al. Manual for the State-Trait Anxiety Inventory. Palo Alto, Consulting Psychologists Press, 1989.

24 Krupp LB, LaRocca NG, Muir-Nash J, et al. The Fatigue Severity Scale. Application to patients with multiple sclerosis and systemic lupus erythematosus. Arch Neurol 1989; 46: 1121-1123.

25 Jones PW, Quirk FH, Baveystock CM, et al. A self-complete measure of health status for chronic airflow limitation. The St. George's Respiratory Questionnaire. Am Rev Respir Dis 1992; 145: 1321-1327.

26 Bestall JC, Paul EA, Garrod R, et al. Usefulness of the Medical Research Council (MRC) dyspnoea scale as a measure of disability in patients with chronic obstructive pulmonary disease. Thorax 1999; 54: 581-586.

27 Yorke J, Moosavi SH, Shuldham C, et al. Quantification of dyspnoea using descriptors: development and initial testing of the Dyspnoea-12. Thorax 2010; 65: 21-26.

28 De Peuter S, Lemaigre V, Van Diest I, et al. Illness-specific catastrophic thinking and overperception in asthma. Health Psychol 2008; 27: 93-99.

29 McCracken LM. "Attention" to pain in persons with chronic pain: a behavioral approach. Behav Ther 1997; 28: 271-284.

30 Carver CS, White TL. Behavioral inhibition, behavioral activation, and affective responses to impending reward and punishment: the BIS/BAS scales. J Pers Soc Psychol 1994; 67: 319-333.

31 Levy ML, Quanjer PH, Booker R, et al. Diagnostic spirometry in primary care: proposed standards for general practice compliant with American Thoracic Society and European Respiratory Society recommendations: a General Practice Airways Group (GPIAG) document, in association with the Association for Respiratory Technology \& Physiology (ARTP) and Education for Health. Prim Care Respir J 2009; 18: 130-147.

32 Wager TD, Davidson ML, Hughes BL, et al. Prefrontal-subcortical pathways mediating successful emotion regulation. Neuron 2008; 59: 1037-1050.

33 Worsley KJ, Evans AC, Marrett S, et al. A three-dimensional statistical analysis for CBF activation studies in human brain. J Cereb Blood Flow Metab 1992; 12: 900-918.

34 Jones PW. St. George's Respiratory Questionnaire: MCID. COPD 2005; 2: 75-79.

35 Parreira VF, Janaudis-Ferreira T, Evans RA, et al. Measurement properties of the incremental shuttle walk test. A systematic review. Chest 2014; 145: 1357-1369.

36 Yorke J, Lloyd-Williams M, Smith J, et al. Management of the respiratory distress symptom cluster in lung cancer: a randomised controlled feasibility trial. Support Care Cancer 2015; 23: 3373-3384.

37 Scioscia G, Blanco I, Arismendi E, et al. Different dyspnoea perception in COPD patients with frequent and infrequent exacerbations. Thorax 2017; 72: 117-121.

38 Leech R, Sharp DJ. The role of the posterior cingulate cortex in cognition and disease. Brain 2014; 137: 12-32.

39 Yang Y, Lueken U, Wittmann A, et al. Neural correlates of individual differences in anxiety sensitivity: an fMRI study using semantic priming. Soc Cogn Affect Neurosci 2016; 11: 1245-1254.

40 Goldin PR, Manber T, Hakimi S, et al. Neural bases of social anxiety disorder: emotional reactivity and cognitive regulation during social and physical threat. Arch Gen Psychiatry 2009; 66: 170-180.

41 Hayen A, Herigstad M, Kelly M, et al. The effects of altered intrathoracic pressure on resting cerebral blood flow and its response to visual stimulation. Neuroimage 2012; 66C: 479-488.

42 Janssens T, De Peuter S, Stans L, et al. Dyspnea perception in COPD: association between anxiety, dyspnea-related fear, and dyspnea in a pulmonary rehabilitation program. Chest 2011; 140: 618-625.

43 Singewald N, Schmuckermair C, Whittle N, et al. Pharmacology of cognitive enhancers for exposure-based therapy of fear, anxiety and trauma-related disorders. Pharmacol Ther 2015; 149: 150-190.

44 Borge CR, Hagen KB, Mengshoel AM, et al. Effects of controlled breathing exercises and respiratory muscle training in people with chronic obstructive pulmonary disease: results from evaluating the quality of evidence in systematic reviews. BMC Pulm Med 2014; 14: 184. 